\title{
Glaucoma Diagnostic Accuracy of Ganglion Cell Inner Plexiform Layer Thickness Comparison with Peripapillary Retinal Nerve Fiber Layer
}

\author{
A.F.Elsawy, H.M.Fayek and E.A.Mahmoud \\ Ophthalmology Dept., Faculty of Medicine, Benha Univ., Benha, Egypt \\ E-Mail: Eman256@gmail.com
}

\begin{abstract}
Glaucoma is an optic neuropathy characterized by progressive loss of retinal ganglion cells (RGCs) and their axons, eventually resulting in visual field loss. As glaucomatous visual misfortune is irreversible, early determination is significant; be that as it may, this can be trying because of enormous between singular variety in ordinary plate appearance, between onlooker contrasts in circle assessment, and absence of affectability of visual field testing because of physiological excess in retinal ganglion cell responsive fields. The reason for this investigation is to decide the symptomatic exhibition of macular ganglion cell-internal plexiform layer (GCIPL) thickness and to contrast it and that of peripapillary retinal nerve fiber layer (RNFL) thickness to segregate typical eyes and eyes with early glaucoma. 20 eyes with early glaucoma (case gathering) and 20 ordinary eyes (control gathering) were joined up with this examination and their RNFL and GCIPL boundaries were estimated utilizing Topcon 3D ghostly space OCT 2000. the GCIPL and RNFL were more slender and the vertical cup-to-circle width proportion was bigger in subjects with glaucoma than in typical subjects. What's more, aggregate and sub-par GCIPL values were the most exact for the analysis of early glaucoma. high hazard gathering and analyzed gathering of glaucoma ought to be catch up with RNFL and GCIPL thickness utilizing OCT.
\end{abstract}

Keywords: Glaucoma, Ganglion cell, Plexiform Layer thickness, Retinal nerve fiber.

\section{Introduction}

Glaucoma is a gathering of eye ailments that bring about harm to the optic nerve and vision misfortune, when vision misfortune has happened, it will be changeless and gradually dynamic. Early location is helpful to stop the movement of the sickness [1].

Hazard factors for glaucoma remember expanded weight for the eye, a family ancestry of the condition, headaches, hypertension, and stoutness [1]. For eye pressures an estimation of more noteworthy than 21 $\mathrm{mmHg}$ is frequently utilized with higher weights prompting a more serious hazard. Be that as it may, some may have high eye pressure for quite a long time and never create harm. Then again, optic nerve harm may happen with ordinary weight, known as should be expected pressure glaucoma. Whenever offered early it is conceivable moderate or stop the movement of sickness with medicine, laser treatment, or medical procedure [2].

Glaucoma has been known as the "quiet hoodlum of sight" in light of the fact that the loss of vision normally happens gradually over a significant stretch of time. Around the world, glaucoma is the seconddriving reason for visual deficiency after waterfalls [3].

Screening for glaucoma is normally proceeded as a major aspect of a standard eye assessment performed by ophthalmologists. Testing for glaucoma ought to incorporate estimations of the intraocular pressure by means of tonometry, front chamber point assessment or gonioscopy, and assessment of the optic nerve to search for any noticeable harm or change in the cup-to-plate proportion and furthermore edge appearance and vascular change. A formal visual field test ought to be performed. The retinal nerve fiber layer can be surveyed with imaging strategies, for example, optical cognizance tomography and examining laser ophthalmoscopy (Heidelberg retinal tomogram) [4].

Glaucomatous harm happens in retinal ganglion cells (RGCs) and their axons, prompting trademark changes in the structure of the optic circle and retinal nerve fiber layer (RNFL). Such basic harm impacts the visual capacity of the glaucoma tolerant. RGC misfortune can't be effectively distinguished on routine fundus assessment with a 90-D focal point. $\mathrm{Be}$ that as it may, considerable interobserver changeability in distinguishing unobtrusive changes makes ophthalmoscopy alone a poor technique for identifying glaucomatous movement. Therefore, glaucoma determination is basically founded on trademark optic circle or peripapillary RNFL (pRNFL) changes and the relating visual field (VF) absconds [3].

Be that as it may, as the auxiliary loss of RGCs regularly goes before the useful glaucomatous VF abandons, different basic imaging gadgets have been created to offer dependable discovery of glaucoma when the condition is at the preperimetric stage. In this viewpoint, it was appeared before the approach of OCT innovation that estimations of retinal thickness at the back post utilizing different methods can dependably recognize early glaucoma and screen its movement [5]. Since glaucoma fundamentally influences retinal ganglion cells and their axons, OCT contemplates have so far generally utilized RNFL thickness estimations to recognize glaucoma and its movement given its high reproducibility] and symptomatic capacity to recognize ordinary and ailing eyes [6]. Different investigations have tried absolute macular thickness for its capacity to 
distinguish glaucoma utilizing past renditions of OCT, yet its separating power has been demonstrated to be lower than that of peripapillary RNFL [7].

Ongoing advances in OCT innovation have empowered increasingly point by point and exact quantitative evaluation of glaucomatous auxiliary changes. For instance, the utilization of calculations for intraretinal division permits separate evaluation of individual retinal layers instead of absolute retinal thickness [8]. A couple of ongoing examinations have indicated that the macular ganglion cell complex (GCC) thickness likewise has a decent glaucoma segregating power that is practically identical to that of the RNFL, perhaps on the grounds that the RNFL, which is remembered for GCC estimations, may impact GCC symptomatic force. [9]

Optical cognizance tomography (OCT), can quantify the thickness of retinal layers, for example, the RNFL. Since its improvement for ophthalmology, this innovation has been overwhelmingly utilized for imaging the macula and macular sicknesses. OCT is progressively utilized in glaucoma appraisal on account of its high picture goals, high reproducibility of estimations, and symptomatic precision. GCC estimations got utilizing SD-OCT have been appeared to bear the cost of preferable demonstrative exactness and repeatability over do add up to macular thickness estimations inferred utilizing either timespace TD-OCT or ghastly area SD-OCT. Additionally, GCC thickness connects emphatically with peripapillary RNFL (pRNFL) thickness [10].

This investigation intended to decide the symptomatic presentation of macular ganglion cellinternal plexiform layer (GCIPL) thickness and to contrast it and that of peripapillary retinal nerve fiber layer (RNFL) thickness to segregate typical eyes and eyes with early glaucoma.

\section{Patients and methods}

This prospective study of glaucoma patients and healthy individuals, at glaucoma clinic of Ophthalmology Department of Benha University Hospital, was conducted to evaluate and compare the utility of ganglion cell complex with peripapillary retinal nerve fiber layer and optic nerve head measurements for detection of localized defects in patients with glaucoma using spectral-domain optical coherence tomography. Twenty eyes with early glaucoma were compared with another twenty normal eyes.

All subjects underwent a full ophthalmic examination including measurement of best corrected visual acuity, subjective refraction, intraocular pressure (IOP) using Goldmann applanation tonometry, slit-lamp examination, gonioscopy, dilated fundus examination with 90D lens and visual field testing with a Humphrey Visual Field Analyzer ( in which mean deviation $<-6 \mathrm{~dB}$ in early glaucoma patients).
Inclusion criteria were ages more than 19 years, best corrected visual acuity of $6 / 12$ or better with a spherical equivalent within $\pm 5 \mathrm{D}$ and a cylinder correction within $\pm 3 \mathrm{D}$. A visual field was defined as reliable if the fixation losses were less than $20 \%$, false-positive rates and false-negative rates were less than $33 \%$ each. Criteria for glaucomatous visual field defect were as follows: glaucoma hemifield test outside normal limits, pattern standard deviation with a $\mathrm{P}$ value $<5 \%$, or a cluster of $<3$ points in the pattern deviation plot in a single hemifield (superior or inferior) with a $\mathrm{P}$ value of $<5 \%$, one of which must have a $\mathrm{P}$ value of $<1 \%$. Any one of the preceding criteria, if repeatable, was considered sufficient evidence of a glaucomatous visual field defect. VF defects had to be repeatable on at least 2 consecutive tests.

Glaucomatous eyes were defined as those with a glaucomatous VF defect confirmed by 2 reliable VF examinations and by the presence of glaucomatous optic disc cupping irrespective of the level of IOP. Glaucomatous optic disc cupping was defined as neuroretinal rim thinning, notching, excavation, or RNFL defect with corresponding VF deficit. Color disc and red-free RNFL images were evaluated independently by 2 observers in a random order and masked fashion, without knowledge of the clinical information. The presence of glaucomatous optic disc cupping was determined by a consensus agreement between the 2 observers.

Patients with glaucoma were excluded if they had a best-corrected visual acuity worse than 6/12, refraction error and spherical equivalent outside that of the inclusion criteria, mean deviation on visual field worse than $6 \mathrm{~dB}$, previous or current vitreoretinal diseases or surgery in the study eye, active infection of the anterior or posterior segment of either eye, or evidence of diabetic retinopathy, macular edema, retinal detachment or epiretinal membrane. Patients with a history of dementia, multiple sclerosis, or a life-threatening or debilitating disease were also excluded. Other exclusion criteria were ongoing or a history of use of a photosensitizing agent, such as verteporfin injection and concomitant use of hydroxychloroquine or chloroquine.

\section{Results}

Forty eyes from forty subjects were examined clinically and had OCT imaging. Twenty eyes with early glaucoma were compaired with twenty eyes of normal subjects. The mean age of all subjects was $43.1+/$ + 14.6 ranging from 20 to 68 years. All of them were female Table (1).

Table (2) shows comparison between case and control group according to age, intraocular pressure (IOP) without medication, spherical equivelant (SE), gonioscopy, best corrected visual acuity (BCVA) $\log$ MAR , mean deviation of visual field (MDVF) and the vertical cup disc ratio (C/D) . 
There was no significant difference in the mean age between the normal control subjects $(41.0+/-$ 12.88 years) and those with early glaucoma (45.2+/16.9 years). The IOP without medication shows significant difference between the two groups ( $P$ value $<0.001)$. Spherical equivalent ( $P$ value 0.23 ) and gonioscopy (P value 0.20 ) were not significantly difference between the two groups. As expected the mean deviation of visual field and vertical cup/disc ratio were significantly difference between the two groups ( $\mathrm{P}$ value $<0.001)$.

Table (1) Demographic characteristics of the study participants.

\begin{tabular}{lc}
\hline variables & Value \\
\hline Age Mean \pm SD(range) & $43.1 \pm 14.6(20.0-68.0)$ \\
Gender $\mathbf{n}(\%)$ & $0(0.0)$ \\
Male & $40(100)$ \\
Female & \\
\hline
\end{tabular}

Table (2) Demographic characteristics of study subjects .

\begin{tabular}{|c|c|c|c|c|c|c|}
\hline & Case group (20) & Control group (20) & St t test & P value & & \\
\hline & Mean & \pm SD & Mean & \pm SD & & \\
\hline Age & 45.2 & 16.19 & 41.0 & 12.88 & 0.91 & 0.37 \\
\hline IOP & 24.4 & 1.79 & 17.4 & 1.6 & 13.03 & $<0.001 * *$ \\
\hline SE & -1.03 & 0.91 & -0.75 & 0.43 & 1.22 & 0.23 \\
\hline \multicolumn{7}{|l|}{ Gonioscopy } \\
\hline+3 & $10(50.0)$ & & $6(30.0)$ & & $X^{2}=1.67$ & 0.20 \\
\hline+4 & $10(50.0)$ & & $14(70.0)$ & & & \\
\hline BCVA $\log M A R$ & 0.2 & 0.11 & 0.06 & 0.09 & 4.27 & $<0.001 * *$ \\
\hline MDVF & -4.9 & 0.85 & -0.63 & 0.14 & 22.11 & $<0.001 * *$ \\
\hline VCDR & 0.72 & 0.10 & 0.56 & 0.08 & 5.47 & $<0.001 * *$ \\
\hline
\end{tabular}

Table (3) shows comparison between the two groups according to retinal nerve fiber layer (RNFL) thickness.

As expected the mean total RNFL thickness was highest in the control group and decreased in case group (normal 104.6um, case 90.0um). There was high significant difference between the two groups ( $\mathrm{P}$ value $<0.001$ ) detected in the total, superior and inferior RNFL thickness.

Table (3) Comparison between case and control groups according to RNFL.

\begin{tabular}{lcccccc}
\hline \multirow{2}{*}{ RNFL } & \multicolumn{2}{c}{ Case group (20) } & \multicolumn{2}{c}{ Control group (20) } & St t test & P value \\
\cline { 2 - 7 } & Mean & $\mathbf{\pm S D}$ & Mean & $\mathbf{\pm S D}$ & & \\
\hline Superior & 108.6 & 11.08 & 124.2 & 9.6 & 4.76 & $<0.001^{* *}$ \\
Inferior & 99.9 & 26.01 & 131.8 & 15.6 & 4.7 & $<0.001^{* *}$ \\
Total & 90.0 & 11.59 & 104.6 & 8.02 & 4.63 & $<0.001^{* *}$ \\
\hline
\end{tabular}

Table (4) shows comparison between the two groups according to ganglion cell inner plexiform layer thickness. Patients with glaucoma had significantly thinner GCIPL than normal subjects in all macular sectors. It shows high significant differences in all parameters ( $\mathrm{p}$ value $<0.001$ ).

Table (4) Comparison between case and control groups according to GCIPL.

\begin{tabular}{|c|c|c|c|c|c|c|}
\hline \multirow{2}{*}{ GCIPL } & \multicolumn{2}{|c|}{ Case group (20) } & \multicolumn{2}{|c|}{ Control group (20) } & \multirow{2}{*}{ St t test } & \multirow{2}{*}{$P$ value } \\
\hline & Mean & \pm SD & Mean & \pm SD & & \\
\hline Superior & 63.8 & 5.13 & 73.2 & 3.04 & 7.06 & $<0.001 * *$ \\
\hline Inferior & 63.1 & 4.51 & 73.4 & 2.6 & 8.85 & $<0.001 * *$ \\
\hline Total & 63.5 & 4.21 & 73.1 & 2.73 & 8.55 & $<0.001 * *$ \\
\hline
\end{tabular}

The AUCs of RNFL and GCIPL for detecting glaucoma are presented in Table (5) and Table (6) respectively. The total (1.0), inferior sector (0.995) and superior sector (0.95) showed the best performances among GCIPL parameters. The AUCs of the total was significantly higher than that of other GCIPL parameters. As regard RNFL, the total (0.85), inferior sector (0.845) and superior sector (0.845). 
The AUCs of the total was significantly higher than that of other RNFL parameters. The total RNFL $(\mathrm{AUC}=0.85)$ and total GCIPL $(\mathrm{AUC}=1.0)$ were best able to discriminate between early glaucoma and normal eyes.

Table (5) ROC values between normal and early glaucomatous eyes according to RNFL parameters, and the validity of superior, inferior and total RNFL in prediction of glaucoma.

\begin{tabular}{lccc}
\hline & Sup RNFL & Inf RNFL & Total RNFL \\
\hline AUC & 0.845 & 0.845 & 0.85 \\
Cut off point & 119.5 & 125.5 & 101.5 \\
Sensitivity & 90.0 & 90.0 & 90.0 \\
Specificity & 70.0 & 60.0 & 60.0 \\
PPV & 75.0 & 69.2 & 69.2 \\
NPV & 87.5 & 85.7 & 85.7 \\
\hline
\end{tabular}

Table (6) ROC values between normal and early glaucomatous eyes according to GCIPL parameters, and the validity of superior, inferior and total GCIPL in prediction of glaucoma.

\begin{tabular}{lccc}
\hline & Sup GCIPL & Inf GCIPL & Total GCIPL \\
\hline AUC & 0.95 & 0.995 & 1.0 \\
Cut off point & 69.5 & 69.5 & 69.5 \\
Sensitivity & 90.0 & 100 & 100 \\
Specificity & 80.0 & 90.0 & 100 \\
PPV & 81.8 & 90.9 & 100 \\
NPV & 88.9 & 100 & 100 \\
Accuracy & 85.0 & 95.0 & 100 \\
\hline
\end{tabular}

\section{Discussion}

Our examination was structured with the primary target of assessing the analytic exactness of macular GCIPL and peripapillary RNFL thickness by Topcon 3D OCT-2000 for recognizing early glaucoma. The GCA calculation is another potential glaucoma symptomatic apparatus, and, accordingly, it is significant that its presentation be assessed. The significance of surveying new glaucoma analytic tests is defended by the way that regularly the analysis of glaucoma can be troublesome, especially in beginning periods when ophthalmoscopically recognizable auxiliary changes and practical shortage are not yet self-evident.

Our finding that macular GCIPL and peripapillary RNFL thicknesses, just as estimations of ONH boundaries, were all reliable with auxiliary glaucomatous harm bolsters the theory that loss of macular retinal ganglion cells is related with RNFL diminishing and changes to the ONH geology on the grounds that, anatomically, all axonal strands exuding from retinal ganglion cells in the macula join into the $\mathrm{ONH}$.

In view of the ROC examination, we affirmed that the macular GCIPL and RNFL thickness boundaries had for the most part comparable demonstrative qualities for glaucoma recognition. True to form, we found that the GCIPL and RNFL were more slender and the vertical cup-to-circle distance across proportion was bigger in subjects with glaucoma than in ordinary subjects.

By utilizing custom intraretinal division calculations on time-area OCT gadgets, Ishikawa et al [11] and Tan et al [12] found a factually huge more slender GCIPL in subjects with glaucoma contrasted and typical subjects. As far as we could possibly know, the main accessible reference to GCIPL estimations with ghostly space OCT is by Wang et al, [13] who utilized the 3D OCT 1000 gadget (Topcon Corporation, Tokyo, Japan) and a custom intraretinal division calculation to quantify the GCIPL thickness in 26 ordinary subjects and 26 subjects with glaucoma; their division was performed physically.

In Tan O, Chopra V, Lu AT, et al. study, [14] most macular GCC boundaries estimated with ghastly space OCT demonstrated glaucoma segregation capacity like that of peripapillary normal RNFL estimated with time-area OCT.

Mwanza et al. shown that the capacity of macular GCIPL boundaries to segregate between typical eyes and eyes with early glaucoma is excellent.[15] They revealed that the GCIPL boundaries with the best AUROCs in early glaucoma were the base (AUROC $=0.959$ ), inferotemporal (AUROC $=0.956$ ), and normal (AUROC $=0.935)$ GCIPL. Takayama et al. [16] announced that base GCIPL thickness AUC (0.896) was altogether higher than normal GCIPL thickness $(0.821)$ in early glaucoma.

In our examination, the normal GCIPL had the most noteworthy AUCs for recognizing early glaucoma $(\mathrm{AUC}=1.0)$. The substandard GCIPL shows additionally high $($ AUC $=0.995)$, so was intended to be touchy to central RGC misfortune, 
which was the best boundary for precisely distinguishing early glaucoma in our examination.

Of the GCIPL boundaries in our examination, aggregate and substandard GCIPL values were the most precise for the finding of early glaucoma. Our outcomes are for the most part predictable with past investigations on the presentation of macular OCT GCIPL boundaries for identifying glaucoma, aside from in the base GCIPL boundary which is absent in our examination. We property such inconsistency and scale inclination among instruments to division contrasts in the boundaries because of various light sources and laser camera framework sensor among Cirrus and Topcon OCT.

We tried the affectability and explicitness of the different OCT boundaries for the recognition of glaucomatous change. For identifying early glaucoma, we saw that the macular GCIPL boundaries had commonly higher sensitivities (90 $\%-100 \%)$ than the OCT RNFL boundaries (90\%) at practically identical specificities. Our outcomes recommended that macular GCIPL thickness might be a superior indicative marker for early glaucoma than OCT RNFL thickness, despite the fact that the AUROC contrast was not noteworthy. We guessed that macular GCIPL boundaries have a hypothetical preferred position over OCT RNFL boundaries, on the grounds that early RGC misfortune ordinarily offers ascend to secluded harm in the paracentral zones. In addition, macular GCIPL geography is less factor among ordinary people than other indicatively significant structures, for example, the optic circle and RNFL, [17] which may bring about a predominant analytic precision of macular GCIPL boundaries in the beginning time of glaucoma.

It is additionally critical to remember that conditions, for example, diabetic macular edema and age-related macular degeneration, which are probably going to be comorbidities in the age gathering of subjects remembered for this investigation, may influence the retinal thickness. The way that subjects with these pathologies were barred from the examination may have improved the indicative execution of GCIPL and RNFL thickness contrasted and optic circle geography, which might be a constraint of this investigation. In this manner, consequences of the ebb and flow study may not be generalizable to patients with other simultaneous ailments that influence retinal thickness.

\section{Conclusion}

The ability of macular GCIPL parameters to discriminate normal eyes and eyes with early glaucoma is high and comparable to that of the best peripapillary RNFL and ONH parameters.

\section{References}

[1] N. Akhtar, "Glaucoma: Clinical Presentation and Pathophysiology.”, Vol. 1(1), PP.65- 77, 2016.

[2] A. V Mantravad , N. Vadhar, " Glaucoma
Primary Care," Clin. Off. Pract, Vol. 42(3), PP.437-449, 2015.

[3] T.-W. Kim, U.-C. Park, K. H. Park, "Ability of Stratus OCT to identify localized retinal nerve fiber layer defects in patients with normal standard automated perimetry results," Invest. Ophthalmol. Vis. Sci, Vol. 48(4), PP.1635-1641, 2007.

[4] R. Thomas , R. S. Parikh, "How to assess a patient for glaucoma," Community eye Heal, Vol. 19(59), PP.36, 2006.

[5] P. Brusini, C. Tosoni, F. Miani, "Quantitative mapping of the retinal thickness at the posterior pole in chronic open angle glaucoma," Acta Ophthalmol. Scand, Vol. 78, (S232), PP.42-44, 2000.

[6] J.-C. Mwanza, J. D. Oakley, D. L. Budenz, "Ability of cirrus HD-OCT optic nerve head parameters to discriminate normal from glaucomatous eyes ," Ophthalmology , Vol. 118(2), PP.241-248, 2011.

[7] G. Wollstein, "Optical coherence tomography (OCT) macular and peripapillary retinal nerve fiber layer measurements and automated visual fields," Am. J. Ophthalmol, Vol. 138, (2), PP. 218-225, 2004.

[8] A. L. Loduca, C. Zhang, R. Zelkha, "Thickness mapping of retinal layers by spectral-domain optical coherence tomography," Am. J. Ophthalmol, Vol. 150(6), PP.849-855, 2010.

[9] A. Garas, P. Vargha, G. Hollo, "Diagnostic accuracy of nerve fibre layer, macular thickness and optic disc measurements made with the RTVue-100 optical coherence tomograph to detect glaucoma," Eye, Vol. 25(1), PP.57-65, 2011.

[10]M. Seong, "Macular and peripapillary retinal nerve fiber layer measurements by spectral domain optical coherence tomography in normaltension glaucoma," Invest. Ophthalmol. Vis. Sci, Vol. 51(3), PP.1446-1452, 2010.

[11]H. Ishikawa, D. M. Stein, G. Wollstein, "Macular segmentation with optical coherence tomography," Invest. Ophthalmol. Vis. Sci., Vol. 46(6), PP.2012-2017, 2005.

[12] O. Tan, G. Li, A. T.-H. Lu, , "Mapping of macular substructures with optical coherence tomography for glaucoma diagnosis," Ophthalmology , Vol. 115(6), PP.949-956, 2008.

[13]M. Wang, "Measurement of local retinal ganglion cell layer thickness in patients with glaucoma using frequency-domain optical coherence tomography," Arch. Ophthalmol., Vol. 127(7), PP.875-881, 2009.

[14] O. Tan ,"Detection of macular ganglion cell loss in glaucoma by Fourier-domain optical coherence tomography," Ophthalmology, Vol. 116(12), PP.2305-2314, 2009.

[15] J.-C. Mwanza ,"Glaucoma diagnostic accuracy of ganglion cell-inner plexiform layer thickness: 
comparison with nerve fiber layer and optic nerve head," Ophthalmology, Vol. 119(6), PP.1151-1158, 2012.

[16]K. Takayama, "A novel method to detect local ganglion cell loss in early glaucoma using spectral-domain optical coherence tomography,"
Invest. Ophthalmol. Vis. Sci, Vol. 53, (11), PP.6904-6913, 2012.

[17]C. A. Curcio , K. A. Allen, “ Topography of ganglion cells in human retina," J. Comp. Neurol., Vol. 300(1), PP.5-25, 1990. 